\title{
DIRECT COMPARISON OF LES AND EXPERIMENT OF A SINGLE- PULSE IMPINGING JET
}

\author{
M. Bovo ${ }^{1,2}$, L. Davidson ${ }^{1}$ \\ ${ }^{1}$ Chalmers University of Technology, Dep. of Applied Mechanics, Göteborg, Sweden \\ ${ }^{2}$ Volvo Car Corporation, Powertrain, Göteborg, Sweden
}

\begin{abstract}
Experimental results are commonly used to validate numerical simulations. Often, it is challenging to accurately reproduce the experimental setups in the numerical environment, thereby leaving uncertainty in the validation process. An experiment on impinging jets was designed with boundary conditions well suited for implementation in the numerical environment. In this work, LES was implemented to specifically match the experiment of a single-pulse jet impinging on a surface oriented normally to the jet axis. The experiment was designed primarily to study the thermal effects on the impingement zone with high space-time resolution. The temperature evolution of the impingement target was measured via IR camera. The space-time resolved jet velocity-field was measured with PIV and was used as boundary condition for the simulation. The focus of the LES was to replicate the experiment as faithfully as possible in the virtual environment. LES was run multiple times to allow statistical evaluation of the results, as done in the experimental procedure. High levels of agreement were found between the LES and the experimental results, both from a qualitative and a quantitative point of view. This work could therefore be considered as a successful validation of the LES in the study of this type of flows.
\end{abstract}

\section{Introduction}

Jets are remarkable flows found in many natural events and industrial applications. Interesting reviews of impinging gas-jet can be found in Jambunethan et al. (1992), Viskanta (1993) and, more recently, Weigand and Spring (2011). Impinging jets are known for their high levels of heat transfer. This characteristic is of great interest in many technological applications. This flow has therefore been studied in several configurations with varying geometrical and dynamical parameters. The textbook references for impinging jet flow field and heat transfer are Cooper et al. (1993) and Baughn et al. (1989) respectively. These works were the first to report measurements of impinging jets in a format suitable for comparison and validation of CFD (Computational Fluid Dynamics) models. Since then, a number of experimental and numerical investigations using different techniques and jet configurations were carried out, e.g. Katti and Prabhu (2008), Bovo and Davidson (2013). Impinging jets have particular thermo-fluid characteristics which make them difficult to model with numerical simulations and they are indeed a recommended test-case for turbulence models (Cooper et al. (1993)). For these reasons they are the focus of much research.

In many cases jets are not stationary (i.e. continuous), but they are instead delivered as a pulse. Works on pulsating impinging jets are found in relation to the cooling of electronic components. In these cases, most of the material focuses on periodic pulsations in which each pulse is partially affected by the previous one e.g. Azevedo et al. (1994), Hofmann et al. (2007), Janetzke and Nitsche (2009) and Peng et al. (2010). A review of works on multiple jet impingement is found in Weigand and Spring (2011)

In some cases, the jet pulse is completely independent of the previous ones (single-pulse jet). An example of single-pulse impinging jet is fuel injection in diesel engines. In this application, at every power stroke a spray of fuel is injected into the combustion chamber. The spray rapidly evaporates and self-ignites, creating a complex reacting flow. Understanding of this process is essential in the design of internal combustion engines. The study of fuel sprays has shown that an evaporating-combusting spray shares many features with free-jets (e.g. Siebers (1998)). Eventually the spray results in a jet-like flame impinging on the combustion chamber wall (e.g. Bovo and Rojo (2013)). The combination of high temperatures and high heat-transfer coefficients at the jet's impingement-zone poses a significant technological challenge in modern engine design. While the flow's temperature is associated with the combustion process, which is of primary interest for CFD related to internal combustion engine technology, less focus is found in literature regarding the near-wall flow-phenomena in the combustion chamber, which are associated with the heat transfer phenomena.

Numerical approaches studying the general case of stationary impinging jets are extensively treated in literature with the dual purpose of assessing the ability of numerical simulations to capture the phenomenon (Lien et al. (1996), Angiolettia et al. (2005) and Hofmann et al. (2007)) and of gaining insight on the physical phenomenon itself (Hällqvist (2006), Yang and Tsai (2007)). A work by the authors of this paper collects, repeats and reviews many of these models from an engineering point of view (Bovo and Davidson (2013)). The above study investigated the 
ability of numerical models, in particular LES, to capture the thermal effects of impinging jets at different Reynolds numbers. This continues to be the focus of investigations by several researchers. In this process of enquiry the numerical approach increasingly becomes a valuable tool to gain insight on the physical phenomenon, both with respect to the flow field, as in Hadziabdic and Hanjalic (2008), and to the related heat-transfer effects, as in Uddin et al. (2013).

In this and other related works by the authors, the focus was instead shifted from the general case of stationary impinging jets to that of a single-pulse impinging jet, which is more closely related to internal combustion engine applications.

Jets are complex and interesting phenomena capturing the interest of much research in fluid flows. The particular case of pulsating jets is at the core of the present work. However, the focus of this study resides on the jet-wall thermal interaction and the consequent heat transfer, rather than on the study of flow in the near-wall jet region. The reason for this limitation is the practical limit posed by the experimental apparatus in accurately investigating this region.

\section{Experimental Setup}

An experimental apparatus was devised for reliable and repeatable generation of a pulse-jet with characteristics relevant to the fuel injection process in modern diesel engines. The experiment needed to have clearly defined boundaries suitable for implementation in numerical simulations with minimum uncertainty. Furthermore, it needed to offer easy accessibility for multiple measurements, for both flowfield and jet-wall thermal interaction.

In practice, for the experiment, the pulse-jet was achieved by modifying the nozzle of an injector designed for commercial engines. The injector was fed with pressurized air. The pulse-jet was oriented towards a flat surface at controlled temperature. In internal combustion engines the jet-like flame impinges on surfaces at different angles at a fixed distance. For this reason, in the experiment, the target was mounted on a rotating support at a constant distance. The measurement campaign focused on studying the evolution of the pulse-jet impinging on a flat target at different angles with respect to the jet's axis. A detailed description of the experimental apparatus can be found in (Bovo et al. (2013)). The time-space evolution of the jet's velocity field was measured with PIV (Particle Image Velocimetry). The temperature evolution of the target surface was measured with an IR (Infra-Red) camera. The experimental campaign revealed a number of features of the pulse-jet's impingement. The inclination of the impingement surface was found to have a significant effect on the free-jet's tip penetration and evolution. Furthermore, the experimental data were analysed statistically, showing that the jet presents noticeable pulse-topulse variation. The data published in Bovo et al.
(2013) are also collected in a database available via the internet.

In Bovo and Rojo (2013) an LES campaign was carried out with the intent of replicating the measurement campaign focusing on the thermal effect of the pulse-impinging jet on surfaces oriented at different angles, thus assessing the ability of the numerical method to reproduce the physical event. To minimize the sources of discrepancies between the real and the virtual environment, the boundary conditions of the numerical simulation were defined within the measured flow-field of the free-jet. In so doing, the freejet's velocity measurements could be directly mapped at the simulation inlet. Comparison between the experimental and the numerical results confirmed the ability of LES to well reproduce the real event. The agreement in both time developing flow-field and target surface relative temperature variation was high. Furthermore, LES gave an insight on the physical phenomenon otherwise not available with the sole physical experiment.

Two issues were pointed out in the conclusion of Bovo and Rojo (2013). In the experiment, the target surface was coated with high emissivity paint necessary for the IR measurements. The thermal effect of the paint was not accounted for in the simulations, giving a significant discrepancy on target-surface absolute temperature between measurements and simulations. Another independent issue was related to the statistical relevance of the study. The measurements revealed a significant pulse-to-pulse variation, but the available computational power allowed only for one simulation per target inclination in the available time frame of that work.

The present work approaches the abovementioned two issues. The effect of the high emissivity coating is implemented in the simulation, allowing a comparison of the evolution of the target surface temperature in absolute terms. Furthermore, simulations with the same setup were run ten times to study pulse-to-pulse variation. This allowed for a statistically relevant direct comparison between the experimental and the numerical results. The case chosen was that of the pulse-jet impinging normally on the target, since the experiment indicated this as the case with largest pulse-to-pulse variations as well as the one with the highest levels of heat transfer coefficient.

This is a validation work for the LES approach and is therefore limited to the direct comparison between the available experimental results. The results directly comparable are the free-jet velocity flowfield and the target surface temperature. The comparison is done on a relative, absolute and statistical basis. The work focuses on verifying the ability of LES to capture the physical phenomenon, thus supporting the usage of such a numerical tool for further investigation of this interesting flow. 


\section{The case}

The phenomenon of interest is the transient thermal effect of an isolated, single-pulse, unconfined jet, normally impinging on a flat surface with timing and geometry relevant to direct fuel injection in internal combustion engines.

An experimental apparatus was set up to reliably reproduce the event multiple times. Moreover, the apparatus was designed to allow easy access for measurements of both jet flow-field and thermal effects, respectively with PIV and IR camera. In practice the pulse-jet was achieved by modifying the nozzle of an injector built for commercial engines. The original multi-hole nozzle was cut off and replaced with a purposely built single-hole one. The modified nozzle was sealed by the original injector needle and controlled by a solenoid. The unit was fed with synthetic pressurized air. The final assembly could be easily handled and controlled with an emulator of the engine control unit, thus giving a pulse-jet with timings comparable to those of the real application. The pulse-jet was oriented to a flat target at controlled temperature, heated by electric resistances. A detailed description of the experimental apparatus and setup is presented in Bovo et al. (2013).

The pulse jet is delivered from a $6 \mathrm{~mm}$ long, $1 \mathrm{~mm}$ diameter hole. The nozzle is supplied with air at $10 \mathrm{bar}$ and $20^{\circ} \mathrm{C}$. The injection time is $5 \mathrm{~ms}$. The pulse is delivered in quiescent air at $20^{\circ} \mathrm{C}$ and it is normally oriented to a flat target $40 \mathrm{~mm}$ from the nozzle.

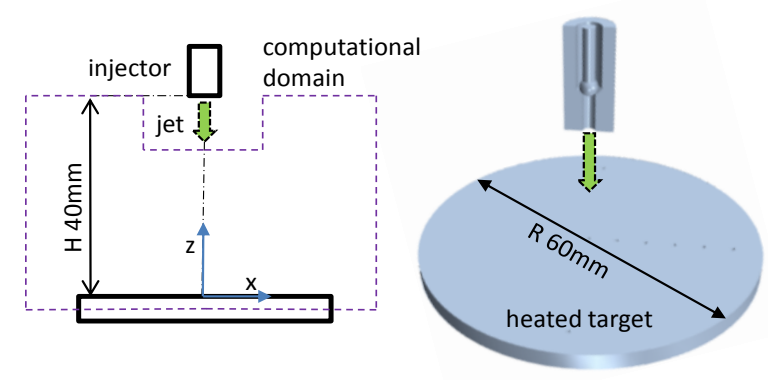

Figure 1: Case overview.

The target is a $60 \mathrm{~mm}$ diameter aluminium block, heated to $150^{\circ} \mathrm{C}$. The target surface is covered with an approximately $0.1 \mathrm{~mm}$ thick coating. The coating is matt black paint with high emissivity, and it is necessary to perform the measurements with the IR camera. This layer of paint plays a significant role in the transient thermal behaviour of the impingement surface.

\section{Modelling methodology}

The computational domain is defined in Figure 2. The fluid is simulated with LES. The WALE (Wall Adapting Local Eddy viscosity) by Nicoud and Ducros (1999) is chosen as sub-grid-scale model. The medium is air, treated as a compressible ideal gas. The flow at the nozzle is under-expanded and becomes subsonic through a series of shock waves. The computational domain does not include the actual nozzle. Instead, in the simulation, the inlet is placed $10 \mathrm{~mm}$ downstream the nozzle where the flow has a Mach number smaller than 0.3 for most of the jet's duration. Inlet conditions are directly taken from measurements. A detailed description of inlet boundary conditions is given in the next section. To resolve conjugated heat transfer, the domain includes the top part of the aluminium target to which a constant temperature boundary is applied. The target coating's thickness is also included and resolved in the computational domain. The paint's properties are: density $990 \mathrm{~kg} / \mathrm{m}^{3}$, specific heat $2000 \mathrm{~J} / \mathrm{kgK}$ and conductivity $2 \mathrm{~W} / \mathrm{mK}$.

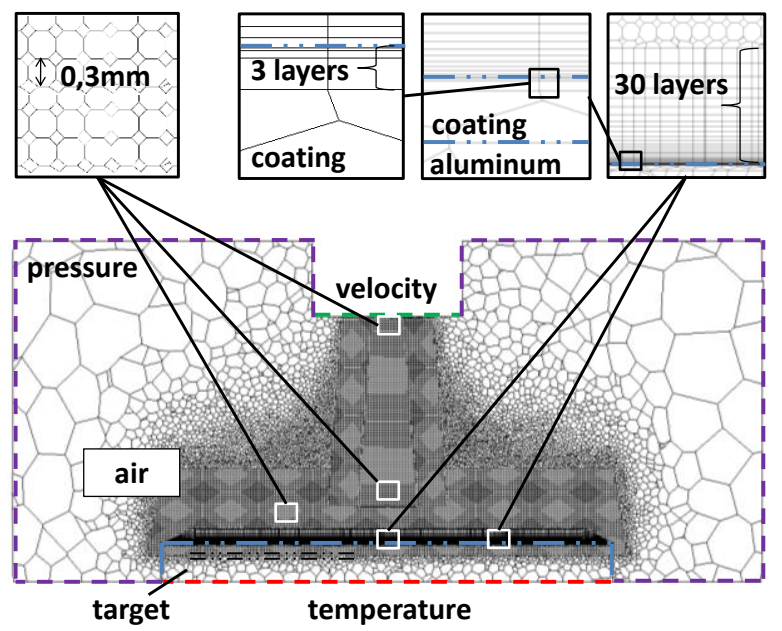

Figure 2: Computational domain, boundary location and mesh details. Velocity, pressure and temperature indicate the position of the different boundary types applied.

The domain is meshed with a fully conformal structure ensuring node-to-node connection at all fluid-solid interfaces. Details of the mesh in different areas can be seen in the zoom-ins in Figure 2. Mesh size refinement is applied to the entire free-jet and wall-jet region. The reference cell size is $0.3 \mathrm{~mm}$. The near wall region is resolved with 30 prism layers, the first layer's thickness is 5e-6mm ensuring $\mathrm{y}^{+}$smaller than one at any location and time. The near-wall cell size in the directions parallel to the wall is in the order of 30 wall units in the impingement region. The paint is resolved with 3 prism layers, with the first layer's thickness, on the fluid side, being $1 \mathrm{e}-6 \mathrm{~mm}$. The mesh has a total of approximately $4.5 \mathrm{e} 6$ cells.

The mesh is constructed with polyhedral elements. This volume-discretization technique is a relatively recent alternative to hexahedral elements, offering two significant advantages. A polyhedral mesh allows for a convenient discretization of complex geometries, often found in real applications. Furthermore, polyhedral meshing allows for a more flexible local cell-size refinement, thus resulting in a smaller total number of cells while still retaining a high mesh-density in regions of interest, as can be appreciated in Figure 2. A more exhaustive evaluation and 
discussion of polyhedral meshing techniques can be found in Peric (2004).

The time-step used in the simulation is $2 \mathrm{e}$ $6 \mathrm{~s}$. With this time-step the maximum Courant number is smaller than 1 in the vast majority of the computational domain with local values as high as approximately 4 in the region closest to the inlet. Although a Courant number smaller than 1 is a requirement to perform accurate LES, the local exceeds are tolerated as they are in the region far from the location of highest interest, i.e. far from the impingement surface.

The simulations were run on a Linux cluster built with Nehalem CPUs (Xeon E5520, 2.27GHz). 4 cores per CPU socket, 8 cores per compute node 24 $48 \mathrm{GiB}$ RAM. Simulations were parallelized on 48 nodes, which was found to be a suitable solution for partitioning and computational load balance. A complete simulation-run lasted approximately one week, limiting the total number of runs executable within the timeframe of this work. The simulations are run using the commercial code Starccm +8.02 .011 by CD-Adapco.

\section{Inlet boundary conditions}

The inlet boundary conditions are based on PIV measurements of the pulse jet and published in Bovo et al. (2013). Two sets of measurements are performed. A measurement campaign is carried out with an acquisition window comprising the entire jettarget area. This setup allowed to follow the entire jet's evolution and to observe the macro-features of the phenomenon, such as the jet's penetration-length in time and the wall-jet formation. Another measurement campaign was conducted with the acquisition window focused on the near-nozzle region. This work aimed to obtain the inlet boundary conditions necessary to properly setup and run CFD simulations attempting to reproduce the measured event.

Figure 3 reports the wall-normal velocity profile average of 100 samples. The profile measured $20 \mathrm{~mm}$ from the nozzle is used for comparison with the simulations' results since this location is within the computational domain. In early phases the pulse-jet is briefly characterized by a gust with velocities noticeably higher than in the later, stationary phase. This effect can be appreciated comparing the profiles in Figure 3 for different locations. Indeed, for data referring to the same time $(0.58 \mathrm{~ms})$, the peak visible in the measurements $20 \mathrm{~mm}$ from the nozzle still carries the effect of the gust, while this effect has already largely disappeared closer to the nozzle $(10 \mathrm{~mm})$.

The measurements taken $10 \mathrm{~mm}$ downstream the nozzle are used as inlet boundary conditions for the numerical simulations. These include both the wallnormal and wall-parallel directions (the latter not shown in the figure). The measured data needed some post-processing to be implemented in the simulations. As can be seen in Figure 3 top, the velocity profile measurements present large errors in the peripheral regions $(\mathrm{x}<-2 \mathrm{~mm}$ and $\mathrm{x}>2 \mathrm{~mm})$. The PIV sys- tem is calibrated to measure jet tip velocity and therefore cannot provide valuable information in areas off the jet's region. This is a well-known limitation of PIV technology that is set up for a limited dynamicrange. The data for locations off the jet region were simply removed (set to 0 ).
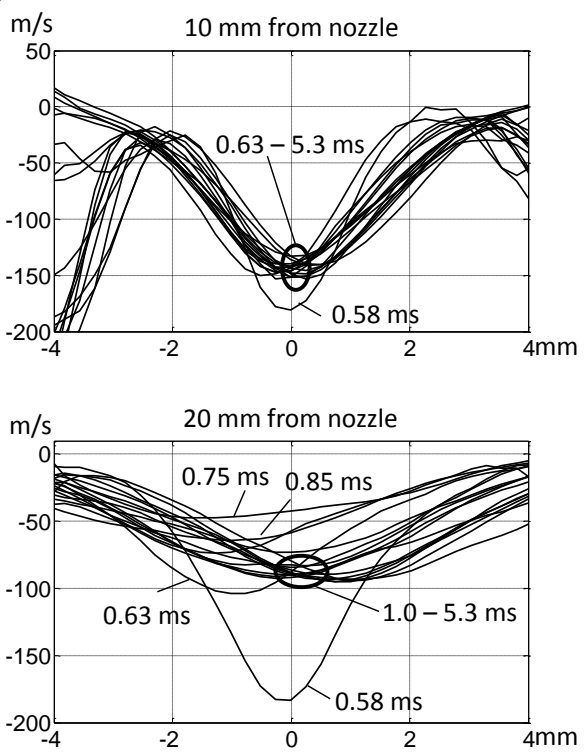

Figure 3: Pulse-jet wall normal velocity profile measured in two locations, as published in Bovo et al. (2013).

The velocity profile was measured on the $2 \mathrm{D}$ plane crossing the nozzle's centre. To provide the inlet conditions for the 3D simulations, the measured profile was revolved around the nozzle's axis to supply information to the entire circular inlet boundary condition. As noticeable in Figure 3, the jet presented minor fluctuations around the nozzle axis. As a result, the velocity profile revolution did not result in a perfectly symmetric shape. At each computational time-step, a new $2 \mathrm{D}$ velocity profile is calculated interpolating between the two measured profiles relevant to the time-step. Thusly, a large-scale turbulent fluctuation with characteristics similar to the one occurring in the real event is introduced in the simulation. This fact can be further elaborated upon, with a number of considerations.

- The actual location of the jet's maximum velocity might not be in the measurements' plane at all times and therefore is not being measured.

- The instantaneous PIV data were not suitable to be used as boundary conditions. The PIV system was limited to one acquisition per jet event, thereby the evolution of a single pulse-jet profile was not available. It was hence necessary to use the average measured profile, a process that de facto further reduces maximum jet velocity. On the other hand, the average measurements show an axial fluctuation indicating a certain repeatability of this phenomenon, which should thereby be included in the simulations. 
- In previous studies it was found that, for jets with nozzle-to-wall distance larger than 6 , the effect of the turbulent fluctuation in the jet's core was overwhelmed by the growth of large turbulent structures in the shear layer. This is believed to be the case also for minor fluctuations of the jet's tip around the jet's axis.

- Synthetic turbulent fluctuations are superimposed to the velocity profiles used as boundary conditions, using the synthetic eddy method described below, to retain the turbulent nature of the flow. These fluctuations further randomize the location of the jet's peak-velocity.

Measurements are always limited in the amount of information they can provide. In this case, it was necessary to extrapolate from the original measurement to supply the simulation's boundary conditions. The extrapolation of information as described above inevitably leads to differences between the real phenomenon and the simulation. Nevertheless, the purpose of the study was repeating the real event in the numerical environment, in order to allow for direct comparison of the results. Therefore, all reasonably possible strategies were implemented to retain the nature of the pulse-jet to be used as boundary conditions from the available experimental data. Nevertheless, it is possible that discrepancies between the real pulse-jet and the profiles used in the simulation may lead to differences between the thermal effects of the two.

At the inlet, the turbulent eddies are superimposed using the synthetic eddy method by Jarrin et al. (2006). Inlet turbulence intensity is set to $20 \%$ and turbulent length scale to $0.15 \mathrm{~mm}$. The characteristics of these fluctuations are derived from statistical elaboration of the instantaneous PIV measurements.

\section{Turbulence and energy modelling}

Turbulence models play a deciding role in the quality of impinging jets simulations. An earlier work by the authors, Bovo and Davidson (2013), focuses on stationary impinging jets. In that work the nature of impinging jets is described. Further, a number of turbulence models are investigated and compared in their prediction of impinging-jet heat transfer. In the abovementioned work it was also shown that the LES modelling approach is able to capture the thermal behaviour of impinging jets.

LES stands for Large Eddy Simulation and, indeed, this method models only small-scale turbulent structures, while it resolves the large scale eddies. Consequently, to properly execute LES, it is necessary to discretize the computational domain with cells small enough to resolve the large turbulent eddies. In LES the Navier-Stokes equation is expressed as:

$$
\rho\left(\frac{\partial \bar{u}_{l}}{\partial t}+\bar{u}_{\jmath} \frac{\partial \bar{u}_{l}}{\partial x_{j}}\right)=-\frac{\partial \bar{p}}{\partial x_{i}}-\frac{\partial \tau_{i j}}{\partial x_{j}}
$$

The turbulent stress tensor $\tau_{\mathrm{ij}}$ is defined as

$$
\tau_{i j}=\rho\left(\overline{u_{\imath} u_{\jmath}}-\overline{u_{i}} \overline{u_{j}}\right)
$$

This term is commonly modelled with a sub-gridmodel based on the Bousinnesq approximation, relating the stress tensor to the local flow by the turbulent viscosity $\mu_{\mathrm{t}}$ and the strain rate. In the study of impinging-jets' heat transfer, the thermal interaction between the jet and the wall is at the core of the phenomena. Therefore, it is necessary to choose a subgrid-model capable of accounting for near wall effects. The WALE (Wall Adapting Local Eddy viscosity) by Nicoud and Ducros (1999) was chosen for this purpose. The definition of turbulent viscosity $\mu_{t}$ in the WALE sub-grid-scale model is

$$
\mu_{t}=\rho \Delta^{2} \frac{\left(S_{i j}^{d} S_{i j}^{d}\right)^{3 / 2}}{\left(\bar{S}_{i j} \bar{S}_{i j}\right)^{5 / 2}+\left(S_{i j}^{d} S_{i j}^{d}\right)^{5 / 4}}
$$

where $\Delta$ is the length scale (or grid filter) defined in terms of the cell volume $V$, locally changing as follows

$$
\Delta=\min \left(\kappa d, C_{w} V^{1 / 3}\right)
$$

$\kappa$ is the von Karman constant, $d$ is cell distance from the wall and $C_{w}=0.544$ is the switch coefficient. The strain tensors are defined as

$$
\begin{gathered}
\bar{S}_{i j}=\frac{1}{2}\left(\frac{\partial \overline{u_{l}}}{\partial x_{j}}+\frac{\partial \overline{u_{j}}}{\partial x_{i}}\right) \\
S_{i j}^{d}=\frac{1}{2}\left(\left(\frac{\partial \bar{u}_{\imath}}{\partial x_{j}}\right)^{2}+\left(\frac{\partial \overline{u_{j}}}{\partial x_{i}}\right)^{2}\right)-\frac{1}{3} \delta_{i j}\left(\frac{\partial \overline{u_{k}}}{\partial x_{k}}\right)^{2}
\end{gathered}
$$

The energy equation is expressed as:

$$
\begin{aligned}
& \frac{\partial(\rho E)}{\partial t}+\frac{\partial\left(u_{j} \rho E\right)}{\partial x_{j}} \\
& =\frac{\partial}{\partial x_{i}}\left(k \frac{\partial T}{\partial x_{i}}\right)+\frac{\partial}{\partial x_{j}}\left(\tau_{i j} u_{j}\right)
\end{aligned}
$$

The turbulent Prandtl number was set to 0.9 and the viscous Prandtl number for air to 0.713 . Which corresponds to a heat conductivity coefficient of $0.0257 \mathrm{~W} / \mathrm{mK}$.

\section{Results: Jet velocity}

In Figure 4 it is possible to follow, side by side, the evolution in time of instantaneous velocity fields for both the measurements and the simulation. Visually, the results show a high level of agreement between LES and the experiment. On a macro-scale, the jet's penetration-length is well captured. Also the size and distribution of the turbulent eddies is well represented. It is possible to identify and compare the large toroidal vortex at the wall-jet's leading edge. Moreover, the size and distribution of smaller eddies 
in the shear layer can be evaluated. The experimental results in Figure 4 do not refer to the same event, but they belong to individual consecutive injections. Indeed, the sampling rate of the PIV system allows to capture only one sample per pulse. It was therefore not possible to follow the flow-field evolution of a single pulse-jet with multiple data acquisitions. In this experiment it was only possible to study the pulse-jet's time-evolution from a statistical point of view. LES, instead, is based on the resolution of the entire time-space evolution of the flow. Therefore, with the numerical approach it is possible to follow the complete evolution of a single event. This is a noteworthy benefit of the numerical simulation approach.
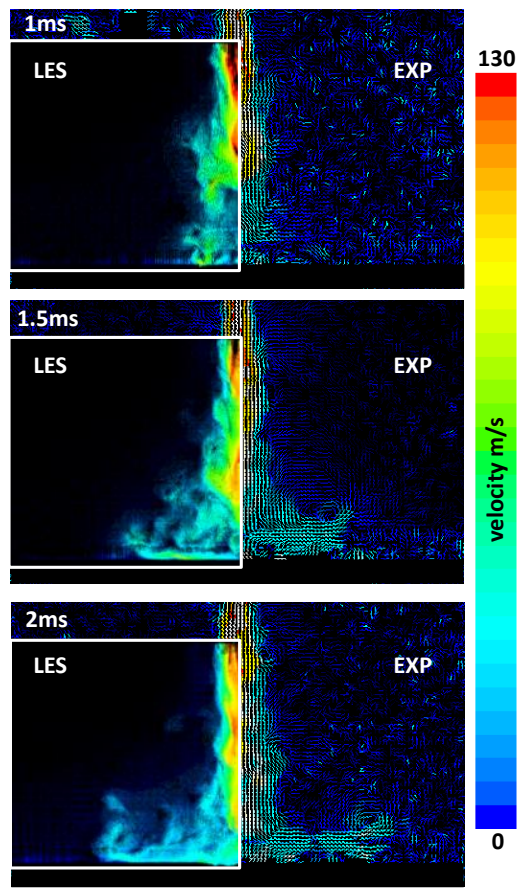

Figure 4: Instantaneous velocity flow field at different times. Comparison between LES and Experiment (PIV).

The LES was run ten times (i.e. ten pulses) and monitored to derive statistically relevant data (see Figure 5). The instantaneous velocity was monitored with four probes shown in the respective figures, two in the core $(x=0 \mathrm{~mm})$ and two in the shear layer $(x=2 \mathrm{~mm})$. One pair of probes was located in the first cell layer adjacent to the inlet boundary, the other pair was positioned at $\mathrm{z}=20 \mathrm{~mm}$.

The largest velocity component is in the wallnormal direction, z. In Figure 5 (top) it is possible to follow the time-evolution in the core of the jet's pulse. Velocity increases rapidly, briefly reaching high values of approximately $350 \mathrm{~m} / \mathrm{s}$. The reference time for all experiments is the injector's open signal. In the graph it is possible to see the delay of the jet's tip in reaching the probes, $10 \mathrm{~mm}$ (red lines) and $20 \mathrm{~mm}$ (blue lines) downstream the actual nozzle, respectively approximately $0.4 \mathrm{~ms}$ and $0.5 \mathrm{~ms}$. Jet veloc- ity at the downstream probe is consistently lower than closer to the inlet, as expected.
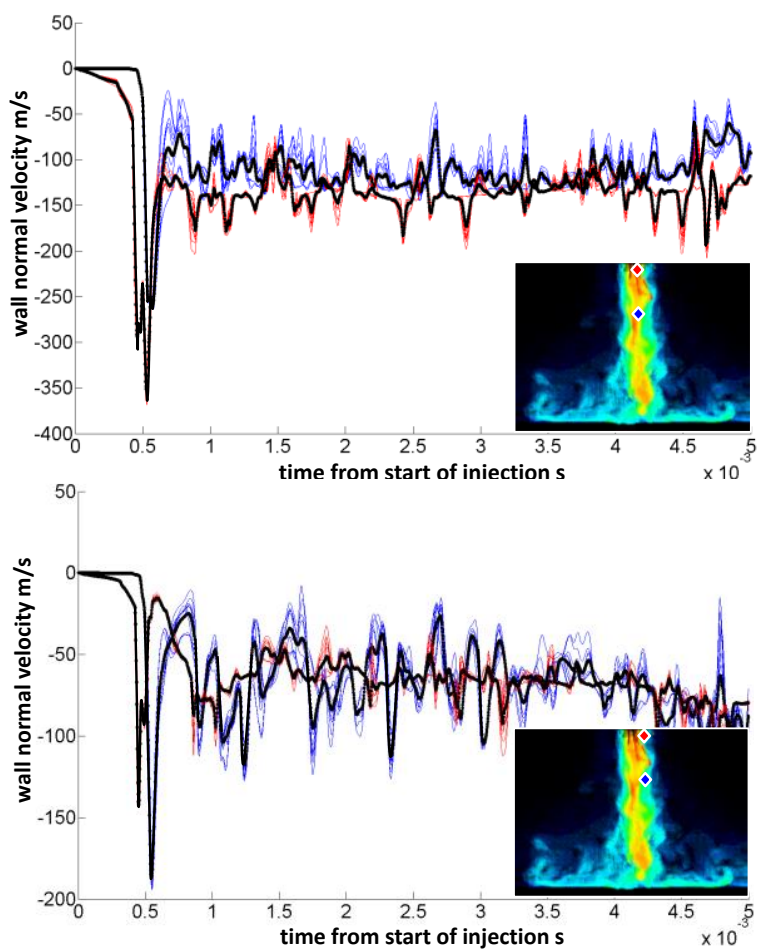

Figure 5: Wall-normal velocity at the jet's core (top) and shear layer (bottom). Log from single pulse in colour, average of ten runs in black.

The velocity fluctuations are the effect of the turbulence created with the synthetic eddy method. Since all the runs were started from the same initial conditions, the model was repeating the same synthetic eddies. The issue was partially solved by changing inlet turbulence intensity and the length scale of the synthetic turbulence by a couple of percentage points. In this respect the model could be further improved.

In the shear layer the velocity is significantly lower than in the core (Figure 5 bottom). At this position it is possible to note that the relative magnitude of the velocity fluctuations is larger compared to the jet's core. In the shear layer, large turbulent structures are generated by the velocity gradient between the jet and the surroundings. These structures grow in size and spread as they move downstream.

The results from the LES and the PIV experiment can be further compared statistically. Figure 6 describes the position and the characteristics of the sampling zone for LES results. Sampling location and size is chosen to match the sampling area of the PIV data reported in Bovo et al. (2013). The area is $1 / 3$ of the distance between the simulation's inlet and the impingement surface. The velocity profiles in wall-normal direction and in radial (or $\mathrm{x}$ ) direction are compared. 


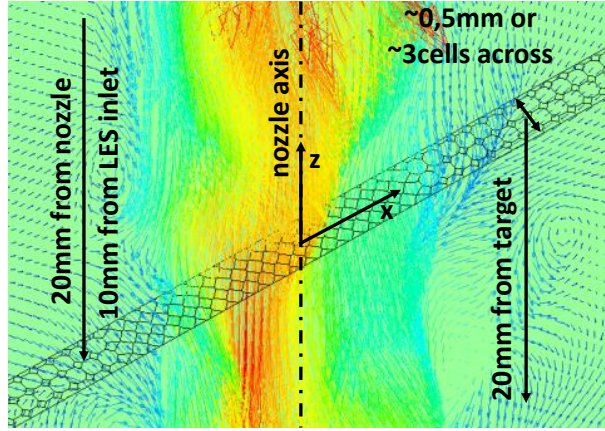

Figure 6: LES mesh corresponding to PIV sampling location for the results in the following figures. LES results velocity vectors are included for clarity. Perspective view.

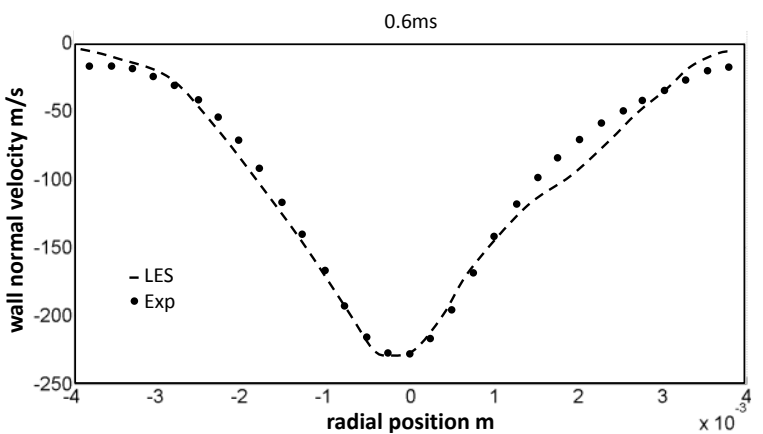

Figure 7: Wall normal velocity profile comparison between LES and PIV during jet early phases.

Figure 7 compares the average of the numerical samples with the PIV measurements at the same time and location. It is worth remembering that PIV results in this plot are the average of 100 PIV pulses. The agreement between the magnitude and the location of jet peak-velocity is high. Note also that velocity magnitude is approximately $200 \mathrm{~m} / \mathrm{s}$ at this time, which is characteristic of the early phases of the pulse jet.

As mentioned earlier, the initial part of the injection is characterized by large velocity fluctuations, in both time and space. Figure 8 presents the velocity in wall-normal direction in later stages, specifically at time $0.9 \mathrm{~ms}$ and $1 \mathrm{~ms}$. In this period the jet begins to stabilize to a velocity magnitude of approximately $100 \mathrm{~m} / \mathrm{s}$. Interesting considerations can be made cross-comparing the results between these times. In other words, comparing PIV at $0.9 \mathrm{~ms}$ with LES at $1 \mathrm{~ms}$ and vice versa. The results seem to match well both magnitude and location of the peak, but they are out of phase. This confirms that in the initial phase the jet is characterized by large fluctuations. Furthermore, it is always important to remember that PIV measurements have the highest accuracy for the specific velocity for which the system is calibrated, with increasing uncertainty for different velocities. For this experiment the PIV system was calibrated for jet peak-velocity.

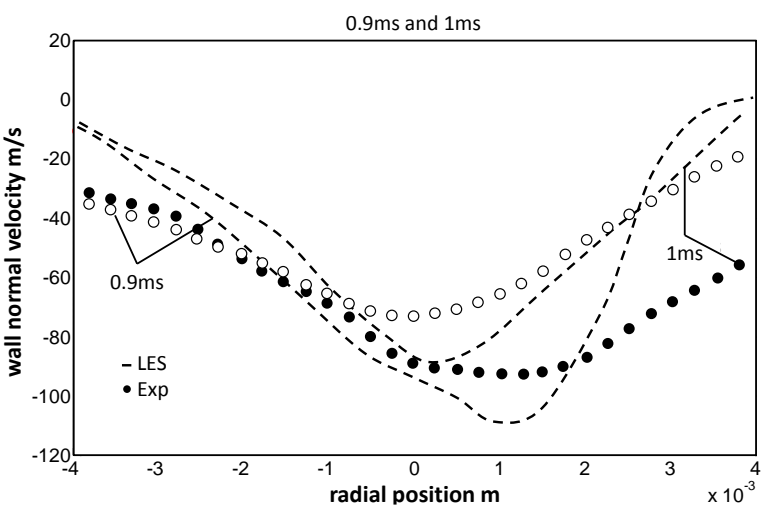

Figure 8: Cross comparison of LES and PIV results in time.

At time approximately $1 \mathrm{~ms}$ the jet reaches a quasi-stationary regime at approximately $90 \mathrm{~m} / \mathrm{s}$. Figure 9 reports the velocity profiles with $1 \mathrm{~ms}$ intervals from $1 \mathrm{~ms}$ to $5 \mathrm{~ms}$. It is possible to notice that the location of the peak-velocity oscillates in time. Both measurements and simulation indicate similar values for the magnitude, location and spreading of jet peakvelocity.

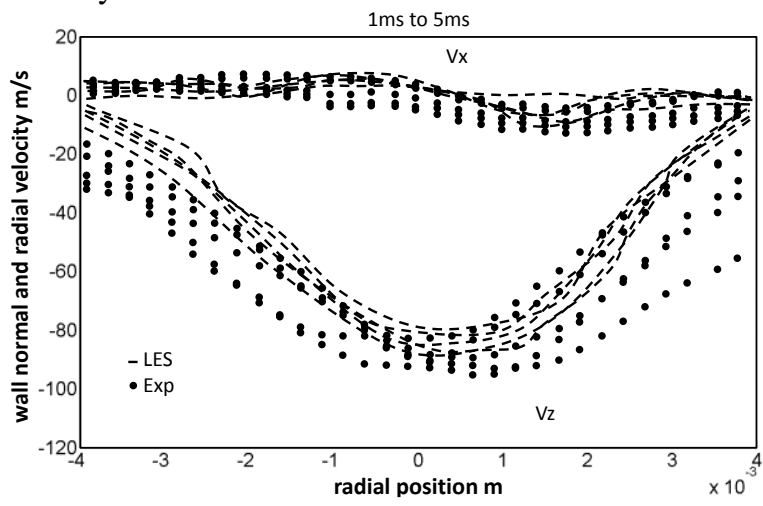

Figure 9: Velocity profile comparison between LES and PIV during jet quasi-stationary period.

\section{Results: Surface temperature evolution}

The present study focuses foremost on the fluidsolid thermal interaction. The temperature evolution of the target surface is hence of primary interest.

Figure 10 (top) shows LES results for the timespace evolution of target surface temperature. In the same figure (bottom) the instantaneous heat transfer coefficient is shown. Here it is possible to appreciate the localized effect of turbulent structures on the near-wall region. In the impingement region the flow shows a smooth transition indicating a viscousdominated boundary layer. Farther away from the impingement zone, the flow transitions to a turbulent regime typical of stationary impinging jets, as found in previous studies by the authors, see Bovo and Davidson (2013).

Figure 10 shows only a wedge of the target, a figure of the complete surface would reveal a nonuniform distribution around the axis of the instantaneous heat transfer coefficient. These large-scale variations are more prominent than the localized fluctuations due to the turbulent structures. This is a conse- 
quence of the fact that the jet is non axis-symmetric, and it oscillates during the injection period, as previously pointed out in the section discussing inlet boundary conditions. The non-symmetric character of the jet is partially repeatable on a pulse-to-pulse basis. This is included in the LES, since inlet boundary conditions are the average of the measured velocity profile. Furthermore, the jet is characterized by random pulse-to-pulse variations due the turbulent nature of the flow. The results are hence further statistically analysed.

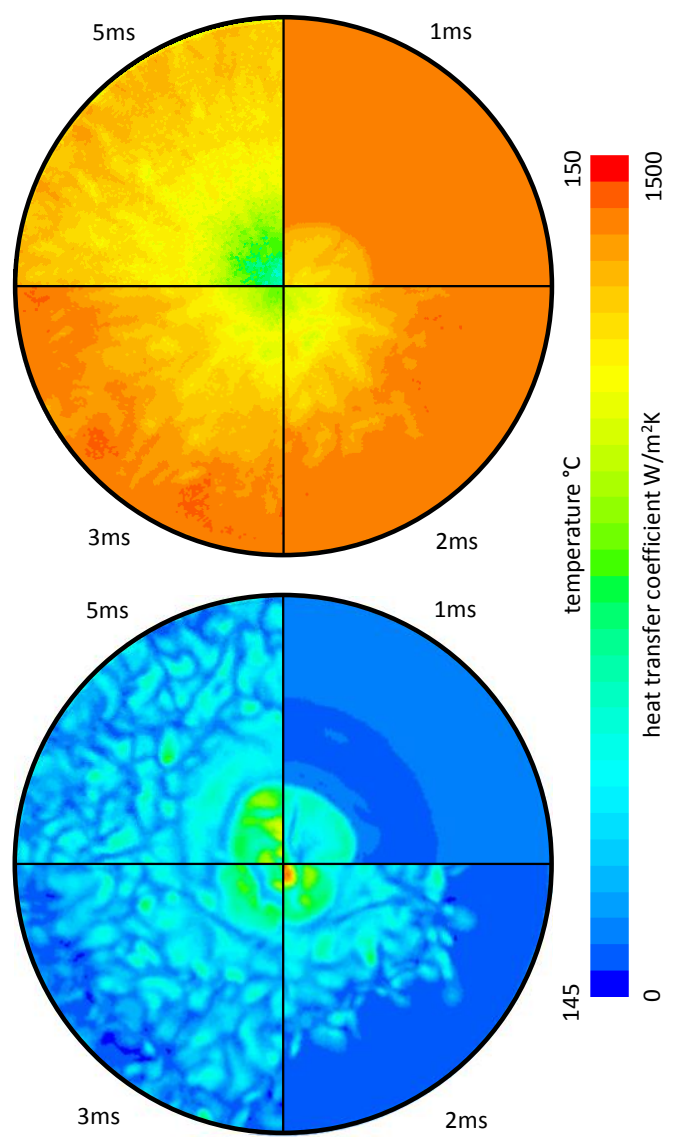

Figure 10: Target surface temperature (top) and heat transfer coefficient (bottom). LES instantaneous results.

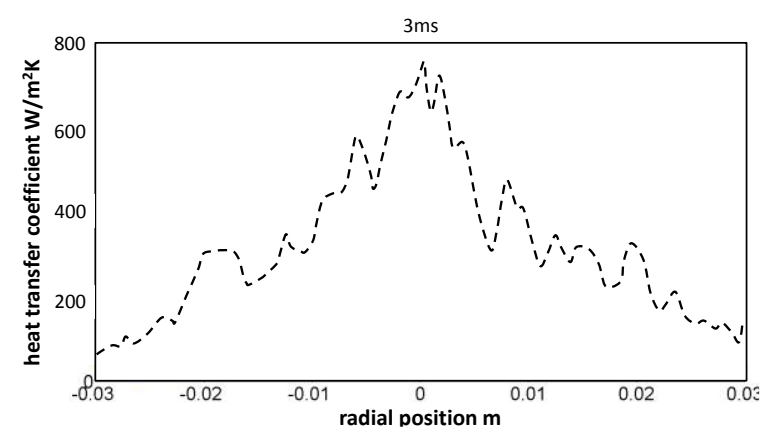

Figure 11: Instantaneous LES heat transfer coefficient (average 10 pulses).

Figure 11 presents the radial distribution of the instantaneous heat-transfer coefficient at time $3 \mathrm{~ms}$. At this time the jet has reached a stationary regime (see velocity in Figure 5). The data report the sampling of all ten runs and their average. The average still presents fluctuations, suggesting that a larger number of samples could be necessary. On the other hand, these fluctuations could be repeatable and caused, to some extent, by the non-randomness of the inlet turbulent fluctuations discussed previously in section 7 .

The highest levels of heat transfer are achieved in the stagnation zone, in accordance with other works in the literature on impinging jet. Instantaneous values, up to $1300 \mathrm{~W} / \mathrm{m}^{2} \mathrm{~K}$, are observed, while the average is approximately $700 \mathrm{~W} / \mathrm{m}^{2} \mathrm{~K}$.

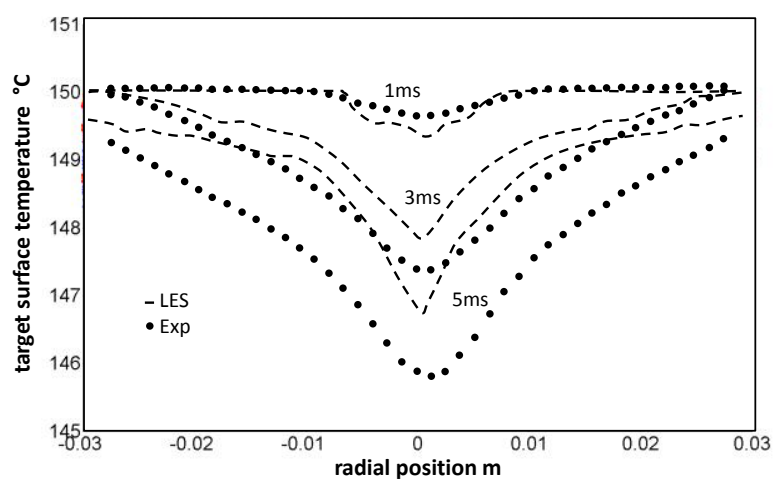

Figure 12: Target surface temperature at different times. Direct comparison between experimental results (average 20 pulses) and LES (average 10 pulses).

The target surface is the only thermal variable available for direct comparison between the LES and the experimental results. In the experiment the temperature is measured with an IR camera on a stripe approximately $1 \mathrm{~mm}$ wide, passing through the target's centre. Data from the corresponding stripe in the LES are reported in Figure 12. The thermal effects of the jet's impingement can be followed in both the experimental and the numerical results. At time $1 \mathrm{~ms}$ the jet has reached the target since a short time and, consequently, the temperature change is limited to the stagnation region, approximately $x<5 \mathrm{~mm}$. At a later stage, time $3 \mathrm{~ms}$, the wall-jet has formed and travelled towards the target's edge. Therefore, its effect on the surface temperature has reached almost $x=30 \mathrm{~mm}$. Finally, at time $5 \mathrm{~ms}$ the jet has had a visible effect on the entire target, including the outermost location.

In Figure 12 even the absolute temperature variation can be directly compared between the LES and the experimental data. The results are similar, with the largest differences in the order of $25 \%$ at time $5 \mathrm{~ms}$. Interestingly, at time $1 \mathrm{~ms}$ the experimental data register a smaller variation than in the LES, while at later times the trend is inverted. There are two potential sources of errors that might explain the discrepancies: one is the measurement technique employed, and another is the lack of accurate material data for the coating. The IR camera's sampling integration time is approximately $0.5 \mathrm{~ms}$ (the total event lasts 
$5 \mathrm{~ms}$ and is captured with 10 samples). This means that, in practice, although the accuracy in temperature measurements is high $(\sim 0.1 \mathrm{~K})$, the time of acquisition is not. Moreover, in initial LES test runs the computed surface-temperature was found to be quite sensitive to the paint's properties and thickness. Unfortunately, the manual painting process could have resulted in non-homogenous paint thickness, which, in turn, could not be measured and reproduced with high accuracy in the numerical model. Furthermore, the thermal material-properties of the paint were not among standard information available with high accuracy from the supplier.

The effect of pulse-to-pulse variation can be studied deriving the standard deviation of the temperature data discussed above and presented in Figure 13. Even for the temperature's standard deviation, the agreement between experimental and LES results is rather high. At time $1 \mathrm{~ms}$ the standard deviation plot presents a very similar step-like shape for both measurements and simulations. The value of the standard deviation gradually increases with the increasing of time, indicating an increasing deviation from the average. The shape of the plots is noticeably narrower for the LES results, which indicates that the fluctuations are more concentrated at the target's centre compared with the experiment; this could be a failure of the model to capture part of the physics. The source of discrepancy might be due to the inlet boundary conditions which do not replicate the real pulse-jet axial fluctuations with sufficient accuracy as explained in section 5. These fluctuations are a potentially important source of the axial spreading seen in the measurements.
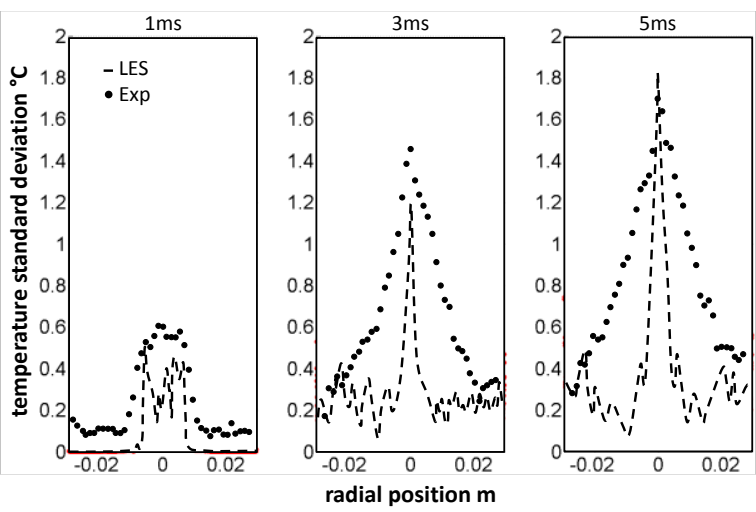

Figure 13: Target temperature standard deviation at different times. Comparison between LES and experiment.

\section{Conclusions}

This work has the primary purpose of assessing LES's ability to capture the thermal effect of an isolate pulse impinging jet. The approach taken consisted in closely replicating an experimental campaign and directly comparing the numerical results with the experimental measurements. This approach allowed a reduction of the differences between the numerical and the physical setup. In particular, the entire com- putational domain was defined within the measured field, thus allowing the use of the actual measurements as inlet boundary conditions for the simulation.

LES was found, in a previous study, to be able to successfully capture the effects of an isolated pulsejet impinging on surfaces at different angles (Bovo and Rojo (2013)). In the present work, the numerical model was updated to include all the components and the respective material properties, thus allowing a direct quantitative comparison of the results. Furthermore, LES was run multiple times, permitting a statistical analysis of the results, as done for the experimental data.

Two sets of data were compared: the velocity flow-field of the jet and the impingement-surface's temperature. The whole velocity field, at different times, was graphically compared. Visual inspection of the results shows that both LES and experimental data captured the size and distribution of the turbulent structures. Moreover, the velocity profiles were extracted from the velocity fields and compared for the same location in the free-jet. The numerical simulation and the experimental data allowed for direct comparison of this variable.

The impingement-surface's temperature is the only variable directly providing information about the thermal interaction between the jet and the target. Temperature evolution was measured with an IR camera along a strip passing through the jet's axis. The measured temperature profile change could be directly compared with simulation results: agreement was found in both shape and absolute values at different times. Furthermore, from the results, the effect of pulse-to-pulse variation was evident. For this reason, the data were further studied statistically and compared in terms of standard deviation. General agreement was found on the absolute values, but somehow less matching in the distribution. Reasons potentially explaining the limited discrepancies between the numerical results and the experimental data were pointed out and should be taken into account by a researcher willing to repeat or extend this work.

The agreement level between experimental data and numerical results provides confidence regarding LES's ability to capture the physics of impinging jets, which was the purpose of this work. When confidence is gained in a numerical approach, it is possible to extend the use of the tool to study similar physical phenomena of interest. Furthermore, simulations permit to follow the event with high time-space resolution. This provides insights on the phenomena which would be otherwise not readily available by solely employing the experimental approach.

\section{Acknowledgements}

The authors thank the Swedish Energy Agency and Volvo Car Corporation for financing the work. 


\section{References}

K. Jambunethan, E. Lai, M. A. Moss and B. L. Button (1992). A review of heat transfer data for single circular jet impingement, Int. J. Heat and Fluid Flow 13 (2): 106-115.

R. Viskanta (1993). Heat transfer to impinging isothermal gas and flame jets. Experimental Thermal and Fluid Science 6:

111-134

B. Weigand, S. Spring (2011). Multiple jet impingement - a review, Heat Transfer Res 42 (2) 101142.

D. Cooper, D. C. Jackson, B. E. Launder and G. X. Liao (1993). Impinging jet studies for turbulence model assessment-I. Flow-field experiments. Int. J. Heat and Mass Transfer 36: 2675-2684.

J. W. Baughn, and S. Shimizu (1989). Heat transfer measurements from a surface with uniform heat flux and an impinging jet, ASME J. Heat Transfer 111: 1097

V. Katti and S. V. Prabhu (2008). Experimental study and theoretical analysis of local heat transfer distribution between smooth flat surface and impinging air jet from a circular straight pipe nozzle, Int. J. Heat Mass Transfer 51: 4480-4495.

M. Bovo, L. Davidson (2013), On the Numerical Modeling of Impinging Jets, Heat Transfer - A Practical Approach, Numerical Heat Transfer, Part A: Applications: An Int. J. of Computation and Methodology 64 (4): 290-316.

L. F. A. Azevedo, B. W. Webb and M. Queiroz (1994) Pulsed Air Jet Impingement Heat Transfer, Experimental Thermal and Fluid Science 8: 206-213

H. M. Hofmann, D. L. Movileanu, M. Kind and H. Martin (2007). Influence of a pulsation on heat transfer and flow structure in submerged impinging jets, Int. J. of Heat and Mass Transfer 50: 36383648 .

T. Janetzke and W. Nitsche (2009). Time resolved investigations on flow field and quasi wall shear stress of an impingement configuration with pulsating jets by means of high speed PIV and a surface hot wire array, Int. J. of Heat and Fluid Flow 30: $877-885$.

X. Peng, Y. Boming, Q. Shuxia, J. P. Hee and S. M. Arun (2010), Turbulent impinging jet heat trans- fer enhancement due to intermittent pulsation, Int. $J$. of Thermal Sciences 49: 1247-1252.

D. Siebers (1998), Liquid-Phase Fuel Penetration in Diesel Sprays, SAE Technical Paper 980809.

M. Bovo and B. Rojo (2013). Single Pulse Jet Impingement on Inclined Surface. Heat Transfer and Flow Field, SAE 13ICE-0063.

F. S. Lien, W. L. Chen, and M. A. Leschziner (1996), Low-Reynolds-Number Eddy-Viscosity Modelling Based on Non-linear StressStrain/Vorticity Relations, Proc. 3rd Symp. on Engineering Turbulence Modelling and Measurements, Crete, Greece.

M. Angiolettia, E. Ninoa, G. Ruoccob (2005), CFD turbulent modelling of jet impingement and its validation by particle image velocimetry and mass transfer measurements, Int. J. of Thermal Sciences 44: 349-356

T. Hällqvist (2006), Large Eddy Simulation of Impinging Jets with Heat Transfer, Dissertation Thesis, KTH Mechanics, Stockholm, Sweden.

Y. Yang and S. Tsai (2007), Numerical study of transient conjugate heat transfer of a turbulent impinging jet. Heat and Mass Transfer 50: 799-807.

M. Hadziabdic and K. Hanjalic (2008), Vortical structures and heat transfer in a round impinging jet. J. of Fluid Mechanics 596: 221-260.

N. Uddin, S. O. Neumann and B. Weigand (2013), LES simulations of an impinging jet: On the origin of the second peak in the Nusselt number distribution, Int. J. Heat Mass Transfer 57: 356-368.

M. Bovo, B. Rojo and M. Golubev (2013), Measurements of a Single Pulse Impinging Jet. A CFD Reference, European Physics J. EFM13. Database:www.tfd.chalmers.se/ lada/projects/jetexp/proright.html

F. Nicoud and F. Ducros (1999). Subgrid-Scale Stress Modelling Based on the Square of the Velocity Gradient Tensor, Flow, Turbulence and Combustion 62: 183-200.

M. Peric (2004), Flow simulation using control volumes of arbitrary Polyhedral shape, ERCOFTAC Bulletin 62, September 2004.

N. Jarrin, S. Benamadouche, D. Laurence and R. Prosser (2006). A synthetic-eddy-method for generating inflowconditions for large eddy simulations, Int. J. of Heat and Fluid Flow 27: 585-593. 